\title{
New Retarded Integral Inequalities with Applications
}

\author{
Ravi P. Agarwal, ${ }^{1}$ Young-Ho Kim, ${ }^{2}$ and S. K. Sen ${ }^{1}$ \\ ${ }^{1}$ Department of Mathematical Sciences, Florida Institute of Technology, 150 West University Boulevard, \\ Melbourne, FL 32901, USA \\ ${ }^{2}$ Department of Applied Mathematics, College of Natural Sciences Changwon National University \\ Changwon, Kyeongnam 641-773, South Korea
}

Correspondence should be addressed to Young-Ho Kim, yhkim@changwon.ac.kr

Received 29 January 2008; Accepted 24 April 2008

Recommended by Yeol Je Cho

Some new nonlinear integral inequalities of Gronwall type for retarded functions are established, which extend the results Lipovan (2003) and Pachpatte (2004). These inequalities can be used as basic tools in the study of certain classes of functional differential equations as well as integral equations. A existence and a uniqueness on the solution of the functional differential equation involving several retarded arguments with the initial condition are also indicated.

Copyright () 2008 Ravi P. Agarwal et al. This is an open access article distributed under the Creative Commons Attribution License, which permits unrestricted use, distribution, and reproduction in any medium, provided the original work is properly cited.

\section{Introduction}

The celebrated Gronwall inequality [1] states that if $u$ and $f$ are nonnegative continuous functions on the interval $[a, b]$ satisfying

$$
u(t) \leq c+\int_{a}^{t} f(s) u(s) d s, \quad t \in[a, b]
$$

for some constant $c \geq 0$, then

$$
u(t) \leq c \exp \left(\int_{a}^{t} f(s) d s\right), \quad t \in[a, b]
$$

Since the inequality (1.2) provides an explicit bound to the unknown function $u$ and hence furnishes a handy tool in the study of solutions of differential equations. Because of its 
fundamental importance, several generalizations and analogous results of the inequality (1.2) have been established over years. Such generalizations are, in general, referred to as Gronwall type inequalities [2-6]. These inequalities provide necessary tools in the study of the theory of differential equations, integral equations, and inequalities of various types. Many authors [2-21] have established several other very useful Gronwall-like integral inequalities. Among these inequalities, the following one (Theorem A) due to Ou-Yang [15] needs specific mention. It is useful in the study of boundedness of certain second-order differential equations.

Theorem A (Ou-Yang [15]). If $u$ and $f$ are nonnegative continuous functions on $[0, \infty)$ such that

$$
u^{2}(t) \leq u_{0}^{2}+2 \int_{0}^{t} f(s) u(s) d s
$$

for all $t \in[0, \infty)$, where $u_{0} \geq 0$ is a constant, then

$$
u(t) \leq u_{0}+\int_{0}^{t} f(s) d s, \quad t \in[0, \infty)
$$

The Ou-Yang inequality prompted researchers to devote considerable time for its generalization and consequent applications [3, 4, 9, 11, 14]. For instance, Lipovan established the following generalization (Theorem B) of Ou-Yang's inequality in the process of establishing a connection between stability and the second law of thermodynamics [14].

Theorem B (Lipovan [14]). Let $u, f$, and $g$ be continuous nonnegative functions on $R_{+}$and $c$ be $a$ nonnegative constant. Also, let $w \in C\left(R_{+}, R_{+}\right)$be a nondecreasing function with $w(u)>0$ on $(0, \infty)$ and $\alpha \in C^{1}\left(R_{+}, R_{+}\right)$be nondecreasing with $\alpha(t) \leq t$ on $R_{+}$. If

$$
u^{2}(t) \leq c^{2}+2 \int_{0}^{\alpha(t)}[f(s) u(s) w(u(s))+g(s) u(s)] d s
$$

for all $t \in R_{+}$, then for $0 \leq t \leq t_{1}$,

$$
\begin{aligned}
& u(t) \leq \Omega^{-1}\left[\Omega\left(c+\int_{0}^{\alpha(t)} g(s) d s\right)+\int_{0}^{\alpha(t)} f(s) d s\right], \\
& \Omega(r)=\int_{1}^{r} \frac{d s}{w(s)}, \quad r>0,
\end{aligned}
$$

$\Omega^{-1}$ is the inverse function of $\Omega$, and $t_{1} \in R_{+}$is chosen so that $\left[\Omega\left(c+\int_{0}^{\alpha(t)} g(s) d s\right)+\int_{0}^{\alpha(t)} f(s) d s\right] \in$ $\operatorname{Dom}\left(\Omega^{-1}\right)$ for all $t \in R_{+}$lying in the interval $0 \leq t \leq t_{1}$.

More recently, Pachpatte established further generalization (Theorem C) of Theorem B as follows [20], which is handy in the study of the global existence of solutions to certain integral equations and functional differential equations.

Theorem C (Pachpatte [20]). Let $u, a_{i}, b_{i} \in C\left(I, R_{+}\right)$and let $\alpha_{i} \in C^{1}(I, I)$ be nondecreasing with $\alpha_{i}(t) \leq t$ on $I$ for $i=1, \ldots, n$. Let $p>1$ and $c \geq 0$ be constants and $w \in C\left(R_{+}, R_{+}\right)$be nondecreasing with $w(u)>0$ on $(0, \infty)$. If for $t \in I$,

$$
u^{p}(t) \leq c+p \sum_{i=1}^{n} \int_{\alpha_{i}\left(t_{0}\right)}^{\alpha_{i}(t)} u(s)\left[a_{i}(s) \psi(u(s))+b_{i}(s)\right] d s,
$$


then for $t_{0} \leq t \leq t_{1}$,

$$
u(t) \leq\left[G^{-1}\left(G(A(t))+(p-1) \sum_{i=1}^{n} \int_{\alpha_{i}\left(t_{0}\right)}^{\alpha_{i}(t)} a_{i}(\sigma) d \sigma\right)\right]^{1 /(p-1)},
$$

where

$$
\begin{aligned}
& G(r)=\int_{r_{0}}^{r} \frac{d s}{w\left(s^{1 /(p-1)}\right)}, \quad r \geq r_{0}>0, \\
& A(t)=c^{(p-1) / p}+(p-1) \sum_{i=1}^{n} \int_{\alpha_{i}\left(t_{0}\right)}^{\alpha_{i}(t)} b_{i}(\sigma) d \sigma,
\end{aligned}
$$

$r_{0}>0$ is arbitrary, $G^{-1}$ is the inverse function of $G$ and $t_{1} \in I$ is so chosen that

$$
G(A(t))+(p-1) \sum_{i=1}^{n} \int_{\alpha_{i}\left(t_{0}\right)}^{\alpha_{i}(t)} a_{i}(\sigma) d \sigma \in \operatorname{Dom}\left(G^{-1}\right) .
$$

The present paper establishes some nonlinear retarded inequalities which extend the foregoing theorems. In addition, it illustrates the use/application of these inequalities.

\section{Main results}

Let $R$ denote the set of real numbers, $R_{+}=[0, \infty)$ and $R_{1}=[1, \infty)$. Also, let $I=\left[t_{0}, T\right)$ be the given subset of $R$. Denote by $C^{i}(M, N)$ the class of all $i$-times continuously differentiable functions defined on the set $M$ to the set $N$ for $i=1,2, \ldots$ and $C^{0}(M, N)=C(M, N)$.

Theorem 2.1. Let $u, f_{i}, g_{i} \in C\left(I, R_{+}\right), i=1, \ldots, n$, and let $\alpha_{i} \in C^{1}(I, I)$ be nondecreasing with $\alpha_{i}(t) \leq t, i=1, \ldots, n$. Suppose that $c \geq 0$ and $q>0$ are constants, $\varphi \in C^{1}\left(R_{+}, R_{+}\right)$is an increasing function with $\varphi(\infty)=\infty$ on $I$, and $\psi(u)$ is a nondecreasing continuous function for $u \in R_{+}$with $\psi(u)>0$ for $u>0$. If

$$
\varphi(u(t)) \leq c+\sum_{i=1}^{n} \int_{\alpha_{i}\left(t_{0}\right)}^{\alpha_{i}(t)} u^{q}(s)\left[f_{i}(s) \psi(u(s))+g_{i}(s)\right] d s
$$

for $t \in I$, then

$$
u(t) \leq \varphi^{-1}\left\{G^{-1}\left[\Psi^{-1}\left(\Psi\left(k\left(t_{0}\right)\right)+\sum_{i=1}^{n} \int_{\alpha_{i}\left(t_{0}\right)}^{\alpha_{i}(t)} f_{i}(s) d s\right)\right]\right\}
$$

for $t \in\left[t_{0}, t_{1}\right)$, where

$$
\begin{aligned}
G(r) & =\int_{r_{0}}^{r} \frac{d s}{\left[\varphi^{-1}(s)\right]^{q}}, \quad r \geq r_{0}>0, \\
\Psi(r) & =\int_{r_{0}}^{r} \frac{d s}{\psi\left[\varphi^{-1}\left(G^{-1}(s)\right)\right]}, \quad r \geq r_{0}>0, \\
k\left(t_{0}\right) & =G(c)+\sum_{i=1}^{n} \int_{\alpha_{i}\left(t_{0}\right)}^{\alpha_{i}(t)} g_{i}(s) d s,
\end{aligned}
$$



that

$G^{-1}$ and $\Psi^{-1}$ denote the inverse functions of $G$ and $\Psi$, respectively, for $t \in I . t_{1} \in I$ is so chosen

$$
\Psi\left(k\left(t_{0}\right)\right)+\sum_{i=1}^{n} \int_{\alpha_{i}\left(t_{0}\right)}^{\alpha_{i}(t)} f_{i}(s) d s \in \operatorname{Dom}\left(\Psi^{-1}\right) .
$$

Proof. Assume that $c>0$. Define a function $z(t)$ by the right-hand side of (2.1). Clearly, $z(t)$ is nondecreasing, $u(t) \leq \varphi^{-1}(z(t))$ for $t \in I$ and $z\left(t_{0}\right)=c$. Differentiating $z(t)$ we get

$$
\begin{aligned}
z^{\prime}(t) & =\sum_{i=1}^{n}\left[u\left(\alpha_{i}(t)\right)\right]^{q}\left[f_{i}\left(\alpha_{i}(t)\right) \psi\left(u\left(\alpha_{i}(t)\right)\right)+g_{i}\left(\alpha_{i}(t)\right)\right] \alpha_{i}^{\prime}(t) \\
& \leq\left[\varphi^{-1}(z(t))\right]^{q} \sum_{i=1}^{n}\left[f_{i}\left(\alpha_{i}(t)\right) \psi\left(\varphi^{-1}\left(z\left(\alpha_{i}(t)\right)\right)\right)+g_{i}\left(\alpha_{i}(t)\right)\right] \alpha_{i}^{\prime}(t) .
\end{aligned}
$$

Using the monotonicity of $\varphi^{-1}$ and $z$, we deduce

$$
\left[\varphi^{-1}(z(t))\right]^{q} \geq\left[\varphi^{-1}\left(z\left(t_{0}\right)\right)\right]^{q}=\left[\varphi^{-1}(c)\right]^{q}>0 .
$$

That is

$$
\frac{z^{\prime}(t)}{\left[\left(\varphi^{-1}(z(t))\right)\right]^{q}} \leq \sum_{i=1}^{n}\left[f_{i}\left(\alpha_{i}(t)\right) \psi\left(\varphi^{-1}\left(z\left(\alpha_{i}(t)\right)\right)\right)+g_{i}\left(\alpha_{i}(t)\right)\right] \alpha_{i}^{\prime}(t) .
$$

Setting $t=s$ in the inequality (2.7), integrating it from $t_{0}$ to $t$, using the function $G$ in the left-hand side, and changing variable in the right-hand side, we obtain

$$
G(z(t)) \leq G(c)+\sum_{i=1}^{n} \int_{\alpha_{i}\left(t_{0}\right)}^{\alpha_{i}(t)}\left[f_{i}(s) \psi\left(\varphi^{-1}(z(s))\right)+g_{i}(s)\right] d s .
$$

From the inequality (2.8), we find

$$
G(z(t)) \leq p(t)+\sum_{i=1}^{n} \int_{\alpha_{i}\left(t_{0}\right)}^{\alpha_{i}(t)} f_{i}(s) \psi\left(\varphi^{-1}(z(s))\right) d s
$$

where

$$
p(t)=G(c)+\sum_{i=1}^{n} \int_{\alpha_{i}\left(t_{0}\right)}^{\alpha_{i}(t)} g_{i}(s) d s .
$$

From the inequality (2.9), we observe that

$$
G(z(t)) \leq p\left(t_{1}\right)+\sum_{i=1}^{n} \int_{\alpha_{i}\left(t_{0}\right)}^{\alpha_{i}(t)} f_{i}(s) \psi\left(\varphi^{-1}(z(s))\right) d s,
$$

for $t \leq t_{1}$. Now, define a function $k(t)$ by the right-hand side of (2.11). Clearly, $k(t)$ is nondecreasing, $z(t) \leq G^{-1}(k(t))$ for $t \in I$ and $k\left(t_{0}\right)=p\left(t_{1}\right)$. Differentiating $k(t)$, we get

$$
k^{\prime}(t)=\sum_{i=1}^{n}\left[f_{i}\left(\alpha_{i}(t)\right) \psi\left(\varphi^{-1}\left(z\left(\alpha_{i}(t)\right)\right)\right)\right] \alpha_{i}^{\prime}(t) \leq \psi\left(\varphi^{-1}\left(G^{-1}(k(t))\right)\right) \sum_{i=1}^{n}\left[f_{i}\left(\alpha_{i}(t)\right)\right] \alpha_{i}^{\prime}(t) .
$$


Ravi P. Agarwal et al.

Using the monotonicity of $\psi, \varphi^{-1}, G^{-1}$, and $k$, we deduce

$$
\frac{k^{\prime}(t)}{\psi\left(\varphi^{-1}\left(G^{-1}(k(t))\right)\right)} \leq \sum_{i=1}^{n}\left[f_{i}\left(\alpha_{i}(t)\right)\right] \alpha_{i}^{\prime}(t)
$$

Setting $t=s$ in the inequality (2.13), integrating it from $t_{0}$ to $t$, using the function $\Psi$ in the left-hand side, and changing variable in the right-hand side, we obtain

$$
\Psi(k(t)) \leq \Psi\left(k\left(t_{0}\right)\right)+\sum_{i=1}^{n} \int_{\alpha_{i}\left(t_{0}\right)}^{\alpha_{i}(t)} f_{i}(s) d s .
$$

From the inequalities (2.11) and (2.14), we conclude that

$$
z(t) \leq G^{-1}\left[\Psi^{-1}\left(\Psi\left(p\left(t_{1}\right)\right)+\sum_{i=1}^{n} \int_{\alpha_{i}\left(t_{0}\right)}^{\alpha_{i}(t)} f_{i}(s) d s\right)\right]
$$

for $t_{0} \leq t \leq t_{1}$. Now a combination of $u(t) \leq \varphi^{-1}(z(t))$ and the last inequality in (2.15) for $t_{1}=t$ produces the required inequality.

If $c=0$ we carry out the above procedure with $\varepsilon>0$ instead of $c$ and subsequently let $\varepsilon \rightarrow 0$. This completes the proof.

For the special case $\varphi(u)=u^{p}(p>q>0$ is a constant), Theorem 2.1 gives the following retarded integral inequality for nonlinear functions.

Corollary 2.2. Let $u, f_{i}, g_{i} \in C\left(I, R_{+}\right), i=1, \ldots, n$, and let $\alpha_{i} \in C^{1}(I, I)$ be nondecreasing with $\alpha_{i}(t) \leq t, i=1, \ldots, n$. Suppose that $c \geq 0$ and $p>q>0$ are constants, and $\psi(u)$ is a nondecreasing continuous function for $u \in R_{+}$with $\psi(u)>0$ for $u>0$. If

$$
u^{p}(t) \leq c+\sum_{i=1}^{n} \int_{\alpha_{i}\left(t_{0}\right)}^{\alpha_{i}(t)} u^{q}(s)\left[f_{i}(s) \psi(u(s))+g_{i}(s)\right] d s
$$

for $t \in I$, then

$$
u(t) \leq\left[\Psi_{0}^{-1}\left(\Psi_{0}\left(k_{1}\left(t_{0}\right)\right)+\frac{p-q}{p} \sum_{i=1}^{n} \int_{\alpha_{i}\left(t_{0}\right)}^{\alpha_{i}(t)} f_{i}(s) d s\right)\right]^{1 /(p-q)}
$$

for $t \in\left[t_{0}, \bar{t}\right)$, where

$$
\begin{aligned}
& \Psi_{0}(r)=\int_{r_{0}}^{r} \frac{d s}{\psi\left(s^{1 /(p-q)}\right)}, \quad r \geq r_{0}>0, \\
& k_{1}\left(t_{0}\right)=c^{(p-q) / p}+\frac{p-q}{p} \sum_{i=1}^{n} \int_{\alpha_{i}\left(t_{0}\right)}^{\alpha_{i}(t)} g_{i}(s) d s,
\end{aligned}
$$

$\Psi_{0}^{-1}$ denotes the inverse function of $\Psi_{0}$ for $t \in I . \bar{t} \in I$ is so chosen that

$$
\Psi_{0}\left(k_{1}\left(t_{0}\right)\right)+\frac{p-q}{p} \sum_{i=1}^{n} \int_{\alpha_{i}\left(t_{0}\right)}^{\alpha_{i}(t)} f_{i}(s) d s \in \operatorname{Dom}\left(\Psi_{0}^{-1}\right) .
$$


Proof. The proof follows by an argument similar to that in the proof of Theorem 2.1 with suitable modification. We omit the details here.

Remark 2.3. When $q=1$, from Corollary 2.2, one derives Theorem C. When $p=2, q=1$, from Corollary 2.2, one derives Theorem B.

Theorem 2.1 can easily be applied to generate other useful nonlinear integral inequalities in more general situations. For example, one has the following result (Theorem 2.4).

Theorem 2.4. Let $u \in C\left(I, R_{1}\right), f_{i}, g_{i} \in C\left(I, R_{+}\right), i=1, \ldots, n$, and let $\alpha_{i} \in C^{1}(I, I)$ be nondecreasing with $\alpha_{i}(t) \leq t, i=1, \ldots, n$. Suppose that $c \geq 1$ is a constant, $\varphi \in C^{1}\left(R_{+}, R_{+}\right)$is an increasing function with $\varphi(\infty)=\infty$ and $\psi_{j}(u), j=1,2$ are nondecreasing continuous functions for $u \in R_{+}$with $\psi_{j}(u)>0$ for $u>0$. If

$$
\varphi(u(t)) \leq c+\sum_{i=1}^{n} \int_{\alpha_{i}\left(t_{0}\right)}^{\alpha_{i}(t)} u^{q}(s)\left[f_{i}(s) \psi_{1}(u(s))+g_{i}(s) \psi_{2}(\log (u(s)))\right] d s
$$

for $t \in I$, then

(i) as the case $\psi_{1}(u) \geq \psi_{2}(\log (u))$,

$$
u(t) \leq \varphi^{-1}\left\{G^{-1}\left[\Psi_{1}^{-1}\left(\Psi_{1}(G(c))+\sum_{i=1}^{n} \int_{\alpha_{i}\left(t_{0}\right)}^{\alpha_{i}(t)}\left[f_{i}(s)+g_{i}(s)\right] d s\right)\right]\right\}
$$

for $t \in\left[t_{0}, t_{1}\right)$,

(ii) as the case $\psi_{1}(u)<\psi_{2}(\log (u))$,

$$
u(t) \leq \varphi^{-1}\left\{G^{-1}\left[\Psi_{2}^{-1}\left(\Psi_{2}(G(c))+\sum_{i=1}^{n} \int_{\alpha_{i}\left(t_{0}\right)}^{\alpha_{i}(t)}\left[f_{i}(s)+g_{i}(s)\right] d s\right)\right]\right\}
$$

for $t \in\left[t_{0}, t_{2}\right)$, where

$$
\Psi_{j}(r)=\int_{r_{0}}^{r} \frac{d s}{\psi_{j}\left(\varphi^{-1}\left(G^{-1}(s)\right)\right)}, \quad r \geq r_{0}>0,
$$

$G^{-1}, \Psi_{j}^{-1}, j=1,2$, denote the inverse functions of $G, \Psi_{j}, j=1,2$, respectively, the function $G(t)$ is as defined in Theorem 2.1 for $t \in I$, and $t_{j} \in I, j=1,2$ are so chosen that

$$
\Psi_{j}(G(c))+\sum_{i=1}^{n} \int_{\alpha_{i}\left(t_{0}\right)}^{\alpha_{i}(t)}\left[f_{i}(s)+g_{i}(s)\right] d s \in \operatorname{Dom}\left(\Psi_{j}^{-1}\right) .
$$

Proof. Let $c>0$. Define a function $z(t)$ by the right-hand side of (2.20). Clearly, $z(t)$ is nondecreasing, $u(t) \leq \varphi^{-1}(z(t))$ for $t \in I$ and $z\left(t_{0}\right)=c$. Differentiating $z(t)$, we get

$$
\begin{aligned}
z^{\prime}(t) & =\sum_{i=1}^{n}\left[u\left(\alpha_{i}(t)\right)\right]^{q}\left[f_{i}\left(\alpha_{i}(t)\right) \psi_{1}\left(u\left(\alpha_{i}(t)\right)\right)+g_{i}\left(\alpha_{i}(t)\right) \psi_{2}\left(\log \left(u\left(\alpha_{i}(t)\right)\right)\right)\right] \alpha_{i}^{\prime}(t) \\
& \leq\left[\varphi^{-1}(z(t))\right]^{q} \sum_{i=1}^{n}\left[f_{i}\left(\alpha_{i}(t)\right) \psi_{1}\left(\varphi^{-1}\left(z\left(\alpha_{i}(t)\right)\right)\right)+g_{i}\left(\alpha_{i}(t)\right) \psi_{2}\left(\log \left(\varphi^{-1}\left(z\left(\alpha_{i}(t)\right)\right)\right)\right)\right] \alpha_{i}^{\prime}(t) .
\end{aligned}
$$


Ravi P. Agarwal et al.

Using the monotonicity of $\varphi^{-1}$ and $z$, we deduce

$$
\left[\varphi^{-1}(z(t))\right]^{q} \geq\left[\varphi^{-1}\left(z\left(t_{0}\right)\right)\right]^{q}=\left[\varphi^{-1}(c)\right]^{q}>0 .
$$

That is

$$
\frac{z^{\prime}(t)}{\left[\left(\varphi^{-1}(z(t))\right)\right]^{q}} \leq \sum_{i=1}^{n}\left[f_{i}\left(\alpha_{i}(t)\right) \psi_{1}\left(\varphi^{-1}\left(z\left(\alpha_{i}(t)\right)\right)\right)+g_{i}\left(\alpha_{i}(t)\right) \psi_{2}\left(\log \left(\varphi^{-1}\left(z\left(\alpha_{i}(t)\right)\right)\right)\right)\right] \alpha_{i}^{\prime}(t) .
$$

Setting $t=s$ in the inequality (2.27), integrating it from $t_{0}$ to $t$, using the function $G$ in the left-hand side, and changing variable in the right-hand side, we obtain

$$
G(z(t)) \leq G(c)+\sum_{i=1}^{n} \int_{\alpha_{i}\left(t_{0}\right)}^{\alpha_{i}(t)}\left[f_{i}(s) \psi_{1}\left(\varphi^{-1}(z(s))\right)+g_{i}(s) \psi_{2}\left(\log \left(\varphi^{-1}(z(s))\right)\right)\right] d s .
$$

When $\psi_{1}(u) \geq \psi_{2}(\log (u))$, from the inequality (2.28), we find

$$
G(z(t)) \leq G(c)+\sum_{i=1}^{n} \int_{\alpha_{i}\left(t_{0}\right)}^{\alpha_{i}(t)}\left[f_{i}(s)+g_{i}(s)\right] \psi_{1}\left(\varphi^{-1}(z(s))\right) d s .
$$

Now, define a function $k(t)$ by the right-hand side of (2.29). Clearly, $k(t)$ is nondecreasing, $z(t) \leq G^{-1}(k(t))$ for $t \in I$ and $k\left(t_{0}\right)=G(c)$. Differentiating $k(t)$, we get

$$
\begin{aligned}
k^{\prime}(t) & =\sum_{i=1}^{n}\left[f_{i}\left(\alpha_{i}(t)\right)+g_{i}\left(\alpha_{i}(t)\right)\right] \psi_{1}\left(\varphi^{-1}\left(z\left(\alpha_{i}(t)\right)\right)\right) \alpha_{i}^{\prime}(t) \\
& \leq \psi_{1}\left(\varphi^{-1}\left(G^{-1}(k(t))\right)\right) \sum_{i=1}^{n}\left[f_{i}\left(\alpha_{i}(t)\right)+g_{i}\left(\alpha_{i}(t)\right)\right] \alpha_{i}^{\prime}(t) .
\end{aligned}
$$

Using the monotonicity of $\psi_{1}, \varphi^{-1}, G^{-1}$, and $k$, we deduce

$$
\frac{k^{\prime}(t)}{\psi_{1}\left(\varphi^{-1}\left(G^{-1}(k(t))\right)\right)} \leq \sum_{i=1}^{n}\left[f_{i}\left(\alpha_{i}(t)\right)\right] \alpha_{i}^{\prime}(t) .
$$

Setting $t=s$ in the inequality (2.31), integrating it from $t_{0}$ to $t$, using the function $\Psi_{1}$ in the left-hand side, and changing variable in the right-hand side, we obtain

$$
\Psi_{1}(k(t)) \leq \Psi_{1}\left(k\left(t_{0}\right)\right)+\sum_{i=1}^{n} \int_{\alpha_{i}\left(t_{0}\right)}^{\alpha_{i}(t)}\left[f_{i}(s)+g_{i}(s)\right] d s .
$$

From the inequality (2.32), we conclude that

$$
z(t) \leq G^{-1}\left[\Psi_{1}^{-1}\left(\Psi_{1}(G(c))+\sum_{i=1}^{n} \int_{\alpha_{i}\left(t_{0}\right)}^{\alpha_{i}(t)}\left[f_{i}(s)+g_{i}(s)\right] d s\right)\right]
$$

for $t \in I$. Now a combination of $u(t) \leq \varphi^{-1}(z(t))$ and the last inequality produces the required inequality in (2.21). 
When $\psi_{1}(u)<\psi_{1}(\log (u))$, from the inequality (2.28), we find

$$
G(z(t)) \leq G(c)+\sum_{i=1}^{n} \int_{\alpha_{i}\left(t_{0}\right)}^{\alpha_{i}(t)}\left[f_{i}(s)+g_{i}(s)\right] \psi_{1}\left(\varphi^{-1}(z(s))\right) d s .
$$

Now, by a suitable application of the process from (2.29) to (2.32) in the inequality (2.34), we conclude that

$$
z(t) \leq G^{-1}\left[\Psi_{2}^{-1}\left(\Psi_{2}(G(c))+\sum_{i=1}^{n} \int_{\alpha_{i}\left(t_{0}\right)}^{\alpha_{i}(t)}\left[f_{i}(s)+g_{i}(s)\right] d s\right)\right]
$$

for $t \in I$. Now a combination of $u(t) \leq \varphi^{-1}(z(t))$ and the last inequality produces the required inequality in (2.22).

If $c=0$, we carry out the above procedure with $\varepsilon>0$ instead of $c$ and subsequently let $\varepsilon \rightarrow 0$. This completes the proof.

For the special case $\varphi(u)=u^{p}(p>q>0$ is a constant), Theorem 2.4 gives the following retarded integral inequality for nonlinear functions.

Corollary 2.5. Let $u \in C\left(I, R_{1}\right), f_{i}, g_{i} \in C\left(I, R_{+}\right), i=1, \ldots, n$, and let $\alpha_{i} \in C^{1}(I, I)$ be nondecreasing with $\alpha_{i}(t) \leq t, i=1, \ldots, n$. Suppose that $c \geq 0$ and $p>q>0$ are constants, and $\psi_{j}(u), j=1,2$ are nondecreasing continuous functions for $u \in R_{+}$with $\psi_{j}(u)>0$ for $u>0$. If

$$
u^{p}(t) \leq c+\sum_{i=1}^{n} \int_{\alpha_{i}\left(t_{0}\right)}^{\alpha_{i}(t)} u^{q}(s)\left[f_{i}(s) \psi_{1}(u(s))+g_{i}(s) \psi_{2}(\log u(s))\right] d s
$$

for $t \in I$, then

(i) as the case $\psi_{1}(u) \geq \psi_{2}(\log (u))$,

$$
u(t) \leq\left[G_{1}^{-1}\left(G_{1}\left(c^{(p-q) / p}\right)+\frac{p-q}{p} \sum_{i=1}^{n} \int_{\alpha_{i}\left(t_{0}\right)}^{\alpha_{i}(t)}\left[f_{i}(s)+g_{i}(s)\right] d s\right)\right]^{1 /(p-q)}
$$

for $t \in\left[t_{0}, t_{1}\right)$,

(ii) as the case $\psi_{1}(u)<\psi_{2}(\log (u))$,

$$
u(t) \leq\left[G_{2}^{-1}\left(G_{2}\left(c^{(p-q) / p}\right)+\frac{p-q}{p} \sum_{i=1}^{n} \int_{\alpha_{i}\left(t_{0}\right)}^{\alpha_{i}(t)}\left[f_{i}(s)+g_{i}(s)\right] d s\right)\right]^{1 /(p-q)}
$$

for $t \in\left[t_{0}, t_{2}\right)$, where $G_{j}^{-1}, j=1,2$, denote the inverse functions of $G_{j}, j=1,2$,

$$
G_{j}(r)=\int_{r_{0}}^{r} \frac{d s}{\psi_{j}\left(s^{1 /(p-q)}\right)}, \quad r \geq r_{0}>0
$$

for $t \in I$, and $t_{j} \in I, j=1,2$, are chosen so that

$$
G_{j}\left(c^{(p-q) / p}\right)+\frac{p-q}{p} \sum_{i=1}^{n} \int_{\alpha_{i}\left(t_{0}\right)}^{\alpha_{i}(t)}\left[f_{i}(s)+g_{i}(s)\right] d s \in \operatorname{Dom}\left(G_{j}^{-1}\right) .
$$


Proof. The proof follows by an argument similar to that in the proof of Theorem 2.4 with suitable modification. We omit the details here.

Theorem 2.1 can easily be applied to generate another useful nonlinear integral inequalities in more general situations. For example, we have the following result (Theorem 2.6).

Theorem 2.6. Let $u, f_{i}, g_{i} \in C\left(I, R_{+}\right), i=1, \ldots, n$, and let $\alpha_{i} \in C^{1}(I, I)$ be nondecreasing with $\alpha_{i}(t) \leq t, i=1, \ldots, n$. Suppose that $c \geq 0$ and $q>0$ are constants, $\varphi \in C^{1}\left(R_{+}, R_{+}\right)$is an increasing function with $\varphi(\infty)=\infty$ on $I$, and $L, M \in C\left(R_{+}^{2}, R_{+}\right)$satisfy

$$
0 \leq L(t, v)-L(t, w) \leq M(t, w)(v-w)
$$

for $t, v, w \in R_{+}$with $v \geq w \geq 0$. If

$$
\varphi(u(t)) \leq c+\sum_{i=1}^{n} \int_{\alpha_{i}\left(t_{0}\right)}^{\alpha_{i}(t)} u^{q}(s)\left[f_{i}(s) L(s, u(s))+g_{i}(s) u(s)\right] d s
$$

for $t \in I$, then

$$
u(t) \leq \varphi^{-1}\left\{G^{-1}\left[\Omega^{-1}\left(\Omega\left(k_{2}\left(t_{0}\right)\right)+\sum_{i=1}^{n} \int_{\alpha_{i}\left(t_{0}\right)}^{\alpha_{i}(t)}\left[f_{i}(s) M(s)+g_{i}(s)\right] d s\right)\right]\right\}
$$

for $t \in\left[t_{0}, t_{1}\right)$, where

$$
\begin{aligned}
\Omega(r) & =\int_{r_{0}}^{r} \frac{d s}{\varphi^{-1}\left(G^{-1}(s)\right)}, \quad r \geq r_{0}>0, \\
k_{2}\left(t_{0}\right) & =G(c)+\sum_{i=1}^{n} \int_{\alpha_{i}\left(t_{0}\right)}^{\alpha_{i}(t)} f_{i}(s) L(s) d s,
\end{aligned}
$$

$G^{-1}$ and $\Omega^{-1}$ denote the inverse function of $G$ and $\Omega$, respectively, the function $G$ is as defined in Theorem 2.1 for $t \in I$ and $t_{1} \in I$ is so chosen that

$$
\Omega\left(k_{2}\left(t_{0}\right)\right)+\sum_{i=1}^{n} \int_{\alpha_{i}\left(t_{0}\right)}^{\alpha_{i}(t)} f_{i}(s) d s \in \operatorname{Dom}\left(\Omega^{-1}\right) .
$$

Proof. Let $c>0$. Define a function $z(t)$ by the right-hand side of (2.42). Clearly, $z(t)$ is nondecreasing, $u(t) \leq \varphi^{-1}(z(t))$ for $t \in I$ and $z\left(t_{0}\right)=c$. Differentiating $z(t)$, we get

$$
\begin{aligned}
z^{\prime}(t) & =\sum_{i=1}^{n}\left[u\left(\alpha_{i}(t)\right)\right]^{q}\left[f_{i}\left(\alpha_{i}(t)\right) L\left(\alpha_{i}(t), u\left(\alpha_{i}(t)\right)\right)+g_{i}\left(\alpha_{i}(t)\right) u\left(\alpha_{i}(t)\right)\right] \alpha_{i}^{\prime}(t) \\
& \leq\left[\varphi^{-1}(z(t))\right]^{q} \sum_{i=1}^{n}\left[f_{i}\left(\alpha_{i}(t)\right) L\left(\alpha_{i}(t), \varphi^{-1}\left(z\left(\alpha_{i}(t)\right)\right)+g_{i}\left(\alpha_{i}(t)\right) \varphi^{-1}\left(z\left(\alpha_{i}(t)\right)\right)\right] \alpha_{i}^{\prime}(t) .\right.
\end{aligned}
$$

Using the monotonicity of $\varphi^{-1}$ and $z$, we deduce

$$
\frac{z^{\prime}(t)}{\left[\left(\varphi^{-1}(z(t))\right)\right]^{q}} \leq \sum_{i=1}^{n}\left[f_{i}\left(\alpha_{i}(t)\right) L\left(\alpha_{i}(t), \varphi^{-1}\left(z\left(\alpha_{i}(t)\right)\right)\right)+g_{i}\left(\alpha_{i}(t)\right) \varphi^{-1}\left(z\left(\alpha_{i}(t)\right)\right)\right] \alpha_{i}^{\prime}(t) .
$$


Setting $t=s$ in the inequality (2.47), integrating it from $t_{0}$ to $t$, using the function $G$ in the left-hand side, and changing variable in the right-hand side, we obtain

$$
G(z(t)) \leq G(c)+\sum_{i=1}^{n} \int_{\alpha_{i}\left(t_{0}\right)}^{\alpha_{i}(t)}\left[f_{i}(s) L\left(s, \varphi^{-1}(z(s))+g_{i}(s) \varphi^{-1}(z(s))\right] d s .\right.
$$

From the inequalities (2.41), (2.48), we find

$$
G(z(t)) \leq G(c)+\sum_{i=1}^{n} \int_{\alpha_{i}\left(t_{0}\right)}^{\alpha_{i}\left(t_{1}\right)} f_{i}(s) L(s) d s+\sum_{i=1}^{n} \int_{\alpha_{i}\left(t_{0}\right)}^{\alpha_{i}(t)}\left[f_{i}(s) M(s)+g_{i}(s)\right] \varphi^{-1}(z(s)) d s,
$$

for $t \leq t_{1}$. Now, define a function $k_{2}(t)$ by the right-hand side of (2.49). Clearly, $k_{2}(t)$ is nondecreasing, $z(t) \leq G^{-1}\left(k_{2}(t)\right)$ for $t \in I$. Differentiating $k_{2}(t)$, we get

$$
\begin{aligned}
k_{2}^{\prime}(t) & =\sum_{i=1}^{n}\left[f_{i}\left(\alpha_{i}(t)\right) M\left(\alpha_{i}(t)\right)+g_{i}\left(\alpha_{i}(t)\right)\right] \varphi^{-1}(z(s)) \alpha_{i}^{\prime}(t) \\
& \leq \varphi^{-1}\left(G^{-1}\left(k_{2}(t)\right)\right) \sum_{i=1}^{n}\left[f_{i}\left(\alpha_{i}(t)\right) M\left(\alpha_{i}(t)\right)+g_{i}\left(\alpha_{i}(t)\right)\right] \alpha_{i}^{\prime}(t) .
\end{aligned}
$$

Using the monotonicity of $\varphi^{-1}, G^{-1}$, and $k_{2}$, we deduce

$$
\frac{k_{2}^{\prime}(t)}{\psi\left(\varphi^{-1}\left(G^{-1}\left(k_{2}(t)\right)\right)\right)} \leq \sum_{i=1}^{n}\left[f_{i}\left(\alpha_{i}(t)\right) M\left(\alpha_{i}(t)\right)+g_{i}\left(\alpha_{i}(t)\right)\right] \alpha_{i}^{\prime}(t) .
$$

Setting $t=s$ in the inequality (2.51), integrating it from $t_{0}$ to $t$, using the function $\Omega$ in the left-hand side, and changing variable in the right-hand side, we obtain

$$
\Omega\left(k_{2}(t)\right) \leq \Omega\left(k_{2}\left(t_{0}\right)\right)+\sum_{i=1}^{n} \int_{\alpha_{i}\left(t_{0}\right)}^{\alpha_{i}(t)}\left[f_{i}(s) M(s)+g_{i}(s)\right] d s .
$$

From the inequalities (2.49) and (2.52), we conclude that

$$
z(t) \leq G^{-1}\left[\Omega^{-1}\left(\Omega\left(k_{2}\left(t_{0}\right)\right)+\sum_{i=1}^{n} \int_{\alpha_{i}\left(t_{0}\right)}^{\alpha_{i}(t)}\left[f_{i}(s) M(s)+g_{i}(s)\right] d s\right)\right]
$$

for $t_{0} \leq t \leq t_{1}$. Now a combination of $u(t) \leq \varphi^{-1}(z(t))$ and the last inequality produces the required inequality in (2.43) for $t_{1}=t$.

If $c=0$, we carry out the above procedure with $\varepsilon>0$ instead of $c$ and subsequently let $\varepsilon \rightarrow 0$. This completes the proof.

For the special case $\varphi(u)=u^{p}(p>q>0$ is a constant), Theorem 2.6 gives the following retarded integral inequality for nonlinear functions.

Corollary 2.7. Let $u, f_{i}, g_{i}$, and $\alpha_{i}$ be as defined in Theorem 2.6. Suppose that $c \geq 0$ and $p>q>0$ are constants, and $L, M \in C\left(R_{+}^{2}, R_{+}\right)$satisfy

$$
0 \leq L(t, v)-L(t, w) \leq M(t, w)(v-w)
$$


for $t, v, w \in R_{+}$with $v \geq w \geq 0$. If

$$
u^{p}(t) \leq c+\sum_{i=1}^{n} \int_{\alpha_{i}\left(t_{0}\right)}^{\alpha_{i}(t)} u^{q}(s)\left[f_{i}(s) L(s, u(s))+g_{i}(s) u(s)\right] d s
$$

for $t \in I$, then

$$
u(t) \leq\left[\Omega_{1}^{-1}\left(\Omega_{1}\left(k_{3}\left(t_{0}\right)\right)+\frac{p-q}{p} \sum_{i=1}^{n} \int_{\alpha_{i}\left(t_{0}\right)}^{\alpha_{i}(t)}\left[f_{i}(s) M(s)+g_{i}(s)\right] d s\right)\right]^{1 /(p-q)}
$$

for $t \in\left[t_{0}, t_{1}\right)$, where

$$
\begin{aligned}
& \Omega_{1}(r)=\int_{r_{0}}^{r} \frac{d s}{s^{1 /(p-q)}}, \quad r \geq r_{0}>0, \\
& k_{3}\left(t_{0}\right)=c^{(p-q) / p}+\frac{p-q}{p} \sum_{i=1}^{n} \int_{\alpha_{i}\left(t_{0}\right)}^{\alpha_{i}(t)} f_{i}(s) L(s) d s,
\end{aligned}
$$

$\Omega_{1}^{-1}$ denotes the inverse function of $\Omega_{1}$ for $t \in I$ and $t_{1} \in I$ is so chosen that

$$
\Omega_{1}\left(k_{3}\left(t_{0}\right)\right)+\frac{p-q}{p} \sum_{i=1}^{n} \int_{\alpha_{i}\left(t_{0}\right)}^{\alpha_{i}(t)}\left[f_{i}(s) M(s)+g_{i}(s)\right] d s \in \operatorname{Dom}\left(\Omega_{1}^{-1}\right) .
$$

Proof. The proof follows by an argument similar to that in the proof of Theorem 2.6 with suitable modification. We omit the details here.

\section{Applications}

We will show that our results are useful in proving the global existence of solutions to certain differential equations with time delay. Consider the functional differential equation involving several retarded arguments with the initial condition:

$$
\begin{gathered}
\varphi^{\prime}(x(t)) x^{\prime}(t)=F\left(t, x\left(t-h_{1}(t)\right), \ldots, x\left(t-h_{n}(t)\right)\right), \quad t \in I, \\
x\left(t_{0}\right)=x_{0},
\end{gathered}
$$

where $x_{0}$ is a constant, $F \in C\left(I \times R^{n}, R\right), h_{i} \in C\left(I, R_{+}\right), i=1, \ldots, n$ be nonincreasing such that $t-h_{i}(t) \geq 0, t-h_{i}(t) \in C^{1}(I, I), h_{i}^{\prime}(t)<1$, and $\varphi \in C^{1}(R, R)$ is an increasing function with $\varphi(|x|) \leq|\varphi(x)|$. The following theorem deals with a bound on the solution of the problem (3.1).

Theorem 3.1. Assume that $F: I \times R^{n} \rightarrow R$ is a continuous function for which there exist continuous nonnegative functions $f_{i}(t), g_{i}(t), i=1, \ldots, n$ for $t \in I$ such that

$$
\left|F\left(t, u_{1}, \ldots, u_{n}\right)\right| \leq \sum_{i=1}^{n}\left|u_{i}\right|^{q}\left[f_{i}(t) \psi\left(\left|u_{i}\right|\right)+g_{i}(t)\right]
$$


where $q>0$ is a constant and $\psi$ is as in Theorem 2.1. Let

$$
Q_{i}=\max _{t \in I} \frac{1}{1-h_{i}^{\prime}(t)}, \quad i=1, \ldots, n
$$

If $x(t)$ is any solution of the problem (3.1), then

$$
|x(t)| \leq \varphi^{-1}\left\{G^{-1}\left[\Psi^{-1}\left(\Psi\left(\bar{k}\left(t_{0}\right)\right)+\sum_{i=1}^{n} \int_{t_{0}-h_{i}\left(t_{0}\right)}^{t-h_{i}(t)} \overline{f_{i}}(\sigma) d \sigma\right)\right]\right\}
$$

for $t \in I$, where $G, \Psi$ are as in Theorem 2.1 and

$$
\bar{k}\left(t_{0}\right)=G\left(\left|\varphi\left(x_{0}\right)\right|\right)+\sum_{i=1}^{n} \int_{t_{0}-h_{i}\left(t_{0}\right)}^{t-h_{i}(t)} \overline{g_{i}}(\sigma) d \sigma
$$

$\overline{f_{i}}(\sigma)=Q_{i} f_{i}\left(\sigma+h_{i}(s)\right), \overline{g_{i}}(\sigma)=Q_{i} g_{i}\left(\sigma+h_{i}(s)\right)$ for $s, \sigma \in I$.

Proof. It is easy to see that the solution $x(t)$ of the problem (3.1) satisfies the equivalent integral equation:

$$
\varphi(x(t))=\varphi\left(x\left(t_{0}\right)\right)+\int_{t_{0}}^{t} F\left(s, x\left(s-h_{1}(s)\right), \ldots, x\left(s-h_{n}(s)\right)\right) d s .
$$

From (3.2) and (3.6), we have

$$
\begin{aligned}
|\varphi(x(t))| & \leq\left|\varphi\left(x\left(t_{0}\right)\right)\right|+\int_{t_{0}}^{t}\left|F\left(s, x\left(s-h_{1}(s)\right), \ldots, x\left(s-h_{n}(s)\right)\right)\right| d s \\
& \leq\left|\varphi\left(x_{0}\right)\right|+\int_{t_{0}}^{t} \sum_{i=1}^{n}\left|x\left(s-h_{i}(s)\right)\right|^{q}\left[f_{i}(t) \psi\left(\left|x\left(s-h_{i}(s)\right)\right|\right)+g_{i}(t)\right] d s
\end{aligned}
$$

for $t, s \in I$. By making the change of variables on the right side of the inequality of (3.7) and rewriting, we have

$$
\varphi(|x(t)|) \leq\left|\varphi\left(x_{0}\right)\right|+\sum_{i=1}^{n} \int_{t_{0}-h_{i}\left(t_{0}\right)}^{t-h_{i}(t)}|x(\sigma)|^{q}\left[\overline{f_{i}}(\sigma) \psi(|x(\sigma)|)+\overline{g_{i}}(\sigma)\right] d \sigma,
$$

where $\overline{f_{i}}(\sigma)=Q_{i} f_{i}\left(\sigma+h_{i}(s)\right), \overline{g_{i}}(\sigma)=Q_{i} g_{i}\left(\sigma+h_{i}(s)\right)$ for $s, \sigma \in I$. Now an immediate application of the inequality established in Theorem 2.1 to the inequality (3.8) yields the result. 
Remark 3.2. Consider the functional differential equation involving several retarded arguments with the initial condition:

$$
\begin{gathered}
p x^{p-1}(t) x^{\prime}(t)=F\left(t, x\left(t-h_{1}(t)\right), \ldots, x\left(t-h_{n}(t)\right)\right), \quad t \in I, \\
x\left(t_{0}\right)=x_{0},
\end{gathered}
$$

where $p>0$ and $x_{0}$ are constants, $F \in C\left(I \times R^{n}, R\right), h_{i} \in C\left(I, R_{+}\right), i=1, \ldots, n$ be nonincreasing such that $t-h_{i}(t) \geq 0, t-h_{i}(t) \in C^{1}(I, I), h_{i}^{\prime}(t)<1$.

Assume that $F: I \times R^{n} \rightarrow R$ is a continuous function for which there exist continuous nonnegative functions $f_{i}(t), g_{i}(t), i=1, \ldots, n$ for $t \in I$ such that

$$
\left|F\left(t, u_{1}, \ldots, u_{n}\right)\right| \leq \sum_{i=1}^{n}\left|u_{i}\right|^{q}\left[f_{i}(t) \psi\left(\left|u_{i}\right|\right)+g_{i}(t)\right]
$$

where $q>0(p>q)$ is a constant and $\psi$ is as in Theorem 2.1. If $x(t)$ is any solution of the problem (3.9), then it satisfies the equivalent integral equation:

$$
x^{p}(t)=x^{p}\left(t_{0}\right)+\int_{t_{0}}^{t} F\left(s, x\left(s-h_{1}(s)\right), \ldots, x\left(s-h_{n}(s)\right)\right) d s .
$$

From (3.10) and (3.11), one has

$$
\begin{aligned}
|x(t)|^{p} & \leq\left|x_{0}\right|^{p}+\int_{t_{0}}^{t}\left|F\left(s, x\left(s-h_{1}(s)\right), \ldots, x\left(s-h_{n}(s)\right)\right)\right| d s \\
& \leq\left|x_{0}\right|^{p}+\int_{t_{0}}^{t} \sum_{i=1}^{n}\left|x\left(s-h_{i}(s)\right)\right|^{q}\left[f_{i}(t) \psi\left(\left|x\left(s-h_{i}(s)\right)\right|\right)+g_{i}(t)\right] d s
\end{aligned}
$$

for $t, s \in I$. By making the change of variables on the right side of (3.12) and rewriting, one has

$$
|x(t)|^{p} \leq\left|x_{0}\right|^{p}+\sum_{i=1}^{n} \int_{t_{0}-h_{i}\left(t_{0}\right)}^{t-h_{i}(t)}|x(\sigma)|^{q}\left[\overline{f_{i}}(\sigma) \psi(|x(\sigma)|)+\overline{g_{i}}(\sigma)\right] d \sigma,
$$

where $\overline{f_{i}}(\sigma)=Q_{i} f_{i}\left(\sigma+h_{i}(s)\right), \overline{g_{i}}(\sigma)=Q_{i} g_{i}\left(\sigma+h_{i}(s)\right)$ for $s, \sigma \in I$. Now an immediate application of the inequality established in Corollary 2.2 to the inequality (3.13) yields

$$
|x(t)| \leq\left[\Psi_{0}^{-1}\left(\Psi_{0}\left(\overline{k_{1}}\left(t_{0}\right)\right)+\frac{p-q}{p} \sum_{i=1}^{n} \int_{t_{0}-h_{i}\left(t_{0}\right)}^{t-h_{i}(t)} \overline{f_{i}}(\sigma) d \sigma\right)\right]^{1 /(p-q)}
$$

for $t \in I$, where $\Psi_{0}$ is as in Corollary 2.2,

$$
\overline{k_{1}}\left(t_{0}\right)=x_{0}^{p-q}+\frac{p-q}{p} \sum_{i=1}^{n} \int_{t_{0}-h_{i}\left(t_{0}\right)}^{t-h_{i}(t)} \overline{g_{i}}(\sigma) d \sigma
$$

$\overline{f_{i}}(\sigma)=Q_{i} f_{i}\left(\sigma+h_{i}(s)\right)$, and $\overline{g_{i}}(\sigma)=Q_{i} g_{i}\left(\sigma+h_{i}(s)\right)$ for $s, \sigma \in I$. 
The following theorem provides a uniqueness on the solution of the problem (3.9).

Theorem 3.3. Assume that $F: I \times R^{3} \rightarrow R$ is a continuous function for which there exist continuous nonnegative functions $f_{i}(t), i=1, \ldots, n$ for $t \in I$ such that

$$
\left|F\left(t, u_{1}, \ldots, u_{n}\right)-F\left(t, v_{1}, \ldots, v_{n}\right)\right| \leq \sum_{i=1}^{n} f_{i}(t)\left|u_{i}^{p}-v_{i}^{p}\right|,
$$

where $p>1$ is a constant, then the problem (3.9) has at most one solution on I.

Proof. Let $x(t)$ and $\bar{x}(t)$ be two distinct solutions of the problem (3.9), one has

$$
x^{p}(t)-\bar{x}^{p}(t)=\int_{t_{0}}^{t}\left[F\left(s, x\left(s-h_{1}(s)\right), \ldots, x\left(s-h_{n}(s)\right)\right)-F\left(s, \bar{x}\left(s-h_{1}(s)\right), \ldots, \bar{x}\left(s-h_{n}(s)\right)\right)\right] d s .
$$

From (3.16) and (3.17), one finds

$$
\left|x^{p}(t)-\bar{x}^{p}(t)\right| \leq \int_{t_{0}}^{t}\left(\sum_{i=1}^{n} f_{i}(s)\left|x^{p}\left(s-h_{i}(s)\right)-\bar{x}^{p}\left(s-h_{i}(s)\right)\right|\right) d s
$$

for $t, s \in I$. By making the change of variables on the right side of (3.18) and rewriting, one has

$$
\left(\left|x^{p}(t)-\bar{x}^{p}(t)\right|^{1 / p}\right)^{p} \leq \sum_{i=1}^{n} \int_{\beta_{i}\left(t_{0}\right)}^{\beta_{i}(t)}\left[\left|x^{p}(\sigma)-\bar{x}^{p}(\sigma)\right|^{1 / p}\right]^{p-1} \bar{f}_{i}(\sigma)\left[\left|x^{p}(\sigma)-\bar{x}^{p}(\sigma)\right|^{1 / p}\right] d \sigma,
$$

where $\beta_{i}(t)=t-h_{i}(t), \overline{f_{i}}(\sigma)=Q_{i} f_{i}\left(\sigma+h_{i}(s)\right)$ for $s, \sigma \in I$. Now when $\psi(u)=u, q=p-1$, a suitable application of the inequality in Corollary 2.2 to the function $\left|x^{p}(t)-\bar{x}^{p}(t)\right|^{1 / p}$ and the inequality (3.19), one concludes that

$$
\left|x^{p}(t)-\bar{x}^{p}(t)\right|^{1 / p} \leq 0
$$

for all $t \in I$. Hence $x(t)=\bar{x}(t)$.

\section{References}

[1] T. H. Gronwall, "Note on the derivatives with respect to a parameter of the solutions of a system of differential equations," Annals of Mathematics, vol. 20, no. 4, pp. 292-296, 1919.

[2] R. P. Agarwal, S. Deng, and W. Zhag, "Generalization of a retarded Gronwall-like inequality and its applications," Applied Mathematics and Computation, vol. 165, no. 3, pp. 599-612, 2005.

[3] D. Bă̌nov and P. Simeonov, Integral Inequalities and Applications, vol. 57 of Mathematics and Its Applications, Kluwer Academic Publishers, Dordrecht, The Netherlands, 1992.

[4] W.-S. Cheung and Q.-H. Ma, "On certain new Gronwall-Ou-Iang type integral inequalities in two variables and their applications," Journal of Inequalities and Applications, vol. 2005, no. 4, pp. 347-361, 2005.

[5] N. -E. Tatar, "An impulsive nonlinear singular version of the Gronwall-Bihari inequality," Journal of Inequalities and Applications, vol. 2006, Article ID 84561, 12 pages, 2006.

[6] H. Ye, J. Gao, and Y. Ding, "A generalized Gronwall inequality and its application to a fractional differential equation," Journal of Mathematical Analysis and Applications, vol. 328, no. 2, pp. 1075-1081, 2007. 
[7] R. Bellman, "The stability of solutions of linear differential equations," Duke Mathematical Journal, vol. 10, pp. 643-647, 1943.

[8] I. Bihari, "A generalization of a lemma of Bellman and its application to uniqueness problems of differential equations," Acta Mathematica Academiae Scientiarum Hungaricae, vol. 7, no. 1, pp. 81-94, 1956.

[9] W.-S. Cheung, "Some new nonlinear inequalities and applications to boundary value problems," Nonlinear Analysis: Theory, Methods \& Applications, vol. 64, no. 9, pp. 2112-2128, 2006.

[10] Y. J. Cho, S. S. Dragomir, and Y. -H. Kim, "On some integral inequalities with iterated integrals," Journal of the Korean Mathematical Society, vol. 43, no. 3, pp. 563-578, 2006.

[11] C. M. Dafermos, "The second law of thermodynamics and stability," Archive for Rational Mechanics and Analysis, vol. 70, no. 2, pp. 167-179, 1979.

[12] S. S. Dragomir and Y.-H. Kim, "On certain new integral inequalities and their applications," Journal of Inequalities in Pure and Applied Mathematics, vol. 3, no. 4, article 65, 8 pages, 2002.

[13] S. S. Dragomir and Y. -H. Kim, "Some integral inequalities for function of two variables," Electronic Journal of Differential Equations, no. 10, pp. 1-13, 2003.

[14] O. Lipovan, "A retarded integral inequality and its applications," Journal of Mathematical Analysis and Applications, vol. 285, no. 2, pp. 436-443, 2003.

[15] L. Ou-Yang, "The boundedness of solutions of linear differential equations $y^{\prime \prime}+A(t) y=0$, " Advances in Mathematics, vol. 3, pp. 409-415, 1957 (Chinese).

[16] B. G. Pachpatte, "Explicit bounds on certain integral inequalities," Journal of Mathematical Analysis and Applications, vol. 267, no. 1, pp. 48-61, 2002.

[17] B. G. Pachpatte, "On some retarded integral inequalities and applications," Journal of Inequalities in Pure and Applied Mathematics, vol. 3, no. 2, article 18, 7 pages, 2002.

[18] B. G. Pachpatte, "On a certain retarded integral inequality and applications," Journal of Inequalities in Pure and Applied Mathematics, vol. 5, no. 1, article 19, 9 pages, 2004.

[19] B. G. Pachpatte, "Inequalities applicable to certain partial differential equations," Journal of Inequalities in Pure and Applied Mathematics, vol. 5, no. 2, article 27, 12 pages, 2004.

[20] B. G. Pachpatte, "On some new nonlinear retarded integral inequalities," Journal of Inequalities in Pure and Applied Mathematics, vol. 5, no. 3, article 80, 8 pages, 2004.

[21] X. Zhao and F. Meng, "On some advanced integral inequalities and their applications," Journal of Inequalities in Pure and Applied Mathematics, vol. 6, no. 3, article 60, 8 pages, 2005. 